\title{
GENERALIZATIONS OF HOPFIAN AND CO-HOPFIAN MODULES
}

YONGDUO WANG

Received 25 September 2004

Let $R$ be a ring and $M$ a left $R$-module. $M$ which satisfies DCC on essential submodules is $\mathrm{GCH}$, and $M$ which satisfies ACC on small submodules is WH. If $M[X]$ is GCH $R[X]$ module, then $M$ is GCH $R$-module. Examples show that a $\mathrm{GCH}$ module need not be co-Hopfian and a WH module need not be Hopfian.

\section{Introduction and preliminaries}

In this paper, all rings are associative with identity and all modules are unital left modules unless otherwise specified.

Let $R$ be a ring and $M$ a module. $N \leq M$ will mean $N$ is a submodule of $M$. A submodule $E$ of $M$ is called essential in $M$ (notation $E \leq_{e} M$ ) if $E \cap A \neq 0$ for any nonzero submodule $A$ of $M$. Dually, a submodule $S$ of $M$ is called small in $M$ (notation $S \ll M$ ) if $M \neq S+T$ for any proper submodule $T$ of $M . M$ is said to be Hopfian (co-Hopfian) in case any surjective (injective) homomorphism is automatically an isomorphism. $M$ is called generalized Hopfian (GH) if any of its surjective endomorphisms has a small kernel. $M$ is called weakly co-Hopfian if any injective endomorphism of $M$ is essential. In this paper, we introduce the concepts of $\mathrm{GCH}$ modules and $\mathrm{WH}$ modules. It is shown that (1) a module $M$ which satisfies DCC on essential submodules is GCH and a module $M$ which satisfies ACC on small submodules is WH; (2) if $M[X]$ is GCH $R[X]$-module, then $M$ is GCH $R$-module. Examples show that a GCH module need not be co-Hopfian and a WH module need not be Hopfian. The notions which are not explained here will be found in [4].

Lemma 1.1 (see $[6,17.3(2)]$ ). Let $K, L$, and $M$ be modules. Then two monomorphisms $f: K \rightarrow L, g: L \rightarrow M$ are essential if and only if $g f$ is essential.

Lemma 1.2 (see $[6,19.3(1)]$ ). Let $K, L$, and $M$ be modules. Then two epimorphisms $f$ : $K \rightarrow L, g: L \rightarrow M$ are small if and only if $g f$ is small.

Lemma 1.3 (see [2, Proposition 5.20]). Suppose that $K_{1} \leq M_{1} \leq M, K_{2} \leq M_{2} \leq M$, and $M=M_{1} \oplus M_{2}$. Then $K_{1} \oplus K_{2} \leq_{e} M_{1} \oplus M_{2}$ if and only if $K_{1} \leq_{e} M_{1}$ and $K_{2} \leq_{e} M_{2}$. 


\section{Generalizations of Hopfian and co-Hopfian modules}

\section{Modules whose essential injective endomorphisms are isomorphic}

Let $M$ be a module. $M$ is said to be a generalized co-Hopfian module (GCH module) if any essential injective endomorphism of $M$ is isomorphic.

Proposition 2.1. Suppose that $M$ satisfies the condition that $N$ is $G C H$ for every proper essential submodule. Then $M$ itself is $G C H$.

Proof. Suppose on the contrary that $M$ is not GCH, then there exists an essential injective homorphism $g: M \rightarrow M$ which is not an isomorphism. Let $N=\operatorname{Im} g$. Then $N \neq M$ and $g$ induces an isomorphism $\bar{g}: M \rightarrow N$. Then $\left.\bar{g}\right|_{N}: N \rightarrow N$ is an essential injective morphism which is not an isomorphism. This is a contradiction since $N$ is a GCH module.

Proposition 2.2. Let $M$ be a GCH module and $K$ be a direct summand of $M$. Then $K$ is GCH.

Proof. Let $M=K \oplus K^{\prime}$ and let $f: K \rightarrow K$ be an essential injection of $K$. $f$ induces an injective endomorphism of $M$, that is, $f \oplus 1_{K^{\prime}}: M \rightarrow M$ with $\left(f \oplus 1_{K^{\prime}}\right)\left(k+k^{\prime}\right)=f(k)+$ $k^{\prime}$. Since $\operatorname{Im}\left(f \oplus 1_{K^{\prime}}\right)=\operatorname{Im} f \oplus K^{\prime} \leq_{e} K \oplus K^{\prime}=M$ by Lemma 1.3, $f \oplus 1_{K^{\prime}}$ is essential. Since $M$ is GCH, $f \oplus 1_{K^{\prime}}$ is isomorphic, and hence $f$ is an isomorphism, as required.

Proposition 2.3. Let $M=M_{1} \oplus M_{2}$ and let $M_{1}, M_{2}$ be invariant submodules under any injection of $M$. Then $M$ is $G C H$ if and only if $M_{1}, M_{2}$ are $G C H$.

Proof. "If" part is clear by Proposition 2.2.

"Only if" part. Let $f: M \rightarrow M$ be an essential injection. Then $\left.f\right|_{M_{i}}: M_{i} \rightarrow M_{i}$ is essential injection. By assumption, $\left.f\right|_{M_{i}}$ is isomorphic. Thus, $f(M)=f\left(M_{1}+M_{2}\right)=f\left(M_{1}\right)+$ $f\left(M_{2}\right)=M_{1}+M_{2}=M$, and hence $f$ is surjective, as desired.

It is easy to know that a module $M$ is co-Hopfian if and only if $M$ is both a weakly co-Hopfian module and a GCH module. In [2], Haghany and Vedadi proved that if DCC holds on nonessential submodules of $M$, then $M$ is weakly co-Hopfian. We also know that Artinian modules are co-Hopfian modules. Thus it is natural that we consider a module with DCC on essential submodules.

Theorem 2.4. Let $M$ be a module with DCC on essential submodules. Then $M$ is GCH.

Proof. Let $f: M \rightarrow M$ be an essential injection of $M$. Then, $\cdots \leq \operatorname{Im} f^{2} \leq \operatorname{Im} f$ is a descending chain on essential submodules of $M$ by Lemma 1.1. Since $M$ satisfies DCC on essential submodules, there exists $n$ such that $\operatorname{Im} f^{n}=\operatorname{Im} f^{n+1}$. For any $m \in M$, since $f^{n}(m) \in \operatorname{Im} f^{n+1}$, there exists $m_{1} \in M$ such that $f^{n}(m)=f^{n+1}\left(m_{1}\right)$. Since $f$ is injective, $m=f\left(m_{1}\right)$. Thus $f$ is surjective, as required.

Lemma 2.5. A quasicontinuous module $M$ is continuous if and only if it is a GCH module.

A continuous module is a quasicontinuous module, but a quasicontinuous module need not be a continuous module. Thus the following result gives a sufficient condition such that a quasicontinuous module is a continuous module.

Corollary 2.6. Let $M$ be a module with DCC on essential submodules. Then $M$ is quasicontinuous if and only if $M$ is continuous. 
Proof. It follows from Theorem 2.4 and Lemma 2.5.

Proposition 2.7. Let $P$ be a property of modules preserved under isomorphism. If a module $M$ has the property $P$ and satisfies DCC on essential submodules with property $P$, then $M$ is $\mathrm{GCH}$.

Proof. Suppose that $M$ is not GCH. Then there exists a proper essential submodule $N_{1}$ of $M$ with $N_{1} \simeq M$. Thus $N_{1}$ is not GCH and enjoys $P$. We have a proper essential submodule $N_{2}$ of $N_{1}$ with $N_{2} \simeq N_{1}$. Clearly, $N_{2}$ is not GCH and satisfies $P$. Repeating, we obtain a strictly descending chain $N_{1}>N_{2}>\cdots$ of proper essential submodules each with property $P$, a contradiction.

Corollary 2.8. If $M$ has DCC on its non-GCH submodules, then $M$ is GCH.

Proof. Suppose not, and let $P$ be the property of being non-GCH. Applying Proposition 2.7, we arrive at a contradiction. Thus $M$ must be GCH.

Example 2.9. A semisimple module $M$ is weakly co-Hopfian if and only if any homogeneous component of $M$ is finitely generated (see [2, Corollary 1.12]). Thus a semisimple module need not be a weakly co-Hopfian module, and hence it is not a co-Hopfian module. However, any semisimple module is GCH.

Let $M$ be a module. The elements of $M[X]$ are formal sums of the form $a_{0}+a_{1} X+$ $\cdots+a_{k} X^{k}$ with $k$ an integer greater than or equal to 0 and $a_{i} \in M$. We denote this sum by $\sum_{i=1}^{k} a_{i} X^{i}\left(a_{0} X^{0}\right.$ is to be understood as the element $\left.a_{0} \in M\right)$. Addition is defined by adding the corresponding coefficients. The $R[X]$-module structure is given by

$$
\left(\sum_{i=0}^{k} \lambda_{i} X^{i}\right) \cdot\left(\sum_{j=0}^{z} a_{j} X^{j}\right)=\sum_{\mu=0}^{k+z} c_{\mu} X^{\mu},
$$

where $c_{\mu}=\Sigma_{i+j=\mu} \lambda_{i} a_{j}$, for any $\lambda_{i} \in R, a_{j} \in M$.

Lemma 2.10 (see [5, Lemma 1.7]). Let $N \leq M$. Then $N$ is essential in $M$ as an R-module if and only if $N[X]$ is essential in $M[X]$ as an $R[X]$-module.

Theorem 2.11. Let $M$ be a module. If $M[X]$ is GCH $R[X]$-module, then $M$ is GCH Rmodule.

Proof. Let $f: M \rightarrow M$ be an essential injective endomorphism of $M$. Then $f[X]: M[X] \rightarrow$ $M[X]$ with $f[X]\left(\Sigma m_{i} X^{i}\right)=\Sigma f\left(m_{i} X^{i}\right)$ is an injective endomorphism of $M[X]$. Next, we will show that $\operatorname{Im} f[X] \leq_{e} M[X]$. It is easy to verify that $\operatorname{Im} f[X]=(\operatorname{Im} f)[X]$. Since $\operatorname{Im} f \leq_{e} M, \operatorname{Im} f[X] \leq_{e} M[X]$ by Lemma 2.10. Thus $f[X]$ is isomorphic by assumption. The surjectivity of $f$ follows by that of $f[X]$. This completes the proof.

\section{Modules whose small surjective endomorphisms are isomorphic}

Let $M$ be a module. $M$ is said to be a weakly Hopfian module (WH module) if any small surjection of $M$ is isomorphic.

Theorem 3.1. Let $M$ be a module. If $M$ satisfies the condition that $M / N$ is $W H$ for every small submodule, $0 \neq N \leq M$. Then $M$ itself is $W H$. 


\section{Generalizations of Hopfian and co-Hopfian modules}

Proof. Suppose on the contrary that $M$ is not WH. Then there exists a small surjection $f$ of $M$ which is not an isomorphism. Let $N=\operatorname{Ker} f$. Then $0 \neq N$ and $f$ induces an isomorphism $\bar{f}: M / N \rightarrow M$. If $\eta: M \rightarrow M / N$ denotes the canonical quotient morphism, then $\eta \bar{f}: M / N \rightarrow M / N$ is a small surjection which is not an isomorphism. This is a contradiction.

Proposition 3.2. Let $M$ be a torsion-free module. Then $M$ is $W H$.

Proof. Let $f: M \rightarrow M$ be any small surjection. Let $0 \neq x \in M \backslash \operatorname{Ker} f$, then $-x \in M \backslash \operatorname{Ker} f$. For any $r \in R, f(x r)=f(x) r$. Since $x \bar{\in} \operatorname{Ker} f, f(x) \neq 0$. By assumption, $f(x) r \neq 0$ and hence $x r \in M \backslash \operatorname{Ker} f$. Thus, $(M \backslash \operatorname{Ker} f) \cup\{0\}$ is a submodule of $M$. Since $(M \backslash \operatorname{Ker} f) \cup$ $\{0\}+\operatorname{Ker} f=M$ and $f$ is a small surjection, $(M \backslash \operatorname{Ker} f) \cup\{0\}=M$ and so $\operatorname{Ker} f=0$, as required.

Proposition 3.3. Let $M$ be $W H$ and $K$ is a direct summand of $M$. Then $K$ is $W H$.

Proof. Since $K$ is a direct summand of $M$, there exists $K^{\prime} \leq M$ such that $M=K \oplus K^{\prime}$. Let $f: K \rightarrow K$ be a surjection with $\operatorname{Ker} f \ll K$, then $f$ induces a small surjection $f \oplus 1_{K^{\prime}}$ : $M \rightarrow M$ with $\left(f \oplus 1_{K^{\prime}}\right)\left(k+k^{\prime}\right)=f(k)+k^{\prime}$ by Lemma 1.2. Since $M$ is WH, $f \oplus 1_{K^{\prime}}$ is isomorphic and hence $f$ is an isomorphism, as desired.

Proposition 3.4. Let $M=M_{1} \oplus M_{2}$ and let $M_{1}, M_{2}$ be invariant submodules under any surjection of $M$. Then $M$ is $W H$ if and only if $M_{1}, M_{2}$ are $W H$.

Proof. "If" part is clear by Proposition 3.3.

"Only if" part. Let $f: M \rightarrow M$ be a small epimorphism, then $\left.f\right|_{M_{i}}: M_{i} \rightarrow M_{i}$ is a small surjection. By assumption, $\left.f\right|_{M_{i}}$ is isomorphic. Let $f\left(m_{1}+m_{2}\right)=0$, then $f\left(m_{1}\right)+$ $f\left(m_{2}\right)=0$ and so $m_{1}=m_{2}=0$. Thus $f$ is injective. This completes the proof.

We know that Noetherian modules are Hopfian modules. It is also easy to know that a module $M$ is Hopfian if and only if $M$ is both a generalized Hopfian module and a WH module. In [1], Ghorbani and Haghany proved that if ACC holds on nonsmall submodules of $M$, then $M$ is generalized Hopfian. Thus it is natural that we consider the following result.

Theorem 3.5. Let $M$ be a module with ACC on small submodules. Then $M$ is WH.

Proof. Let $f: M \rightarrow M$ be a small surjection of $M$. Then $\operatorname{Ker} f \leq \operatorname{Ker} f^{2} \leq \operatorname{Ker} f^{3} \leq \cdots$ is an ascending chain on small submodule of $M$ by Lemma 1.2. Since $M$ satisfies ACC on small submodules, there exists a positive number $n$ such that $\operatorname{Ker} f^{n}=\operatorname{Ker} f^{n+1}$. Next, we will prove that $\operatorname{Ker} f=0$. Let $x \in \operatorname{Ker} f$, then $f(x)=0$. Since $f$ is surjective, there exists $y_{1} \in M$ such that $f\left(y_{1}\right)=x$. Since $f$ is surjective, there exists $y_{2} \in M$ such that $f\left(y_{2}\right)=y_{1}$. Repeating the process, we obtain $y_{n-1} \in M$ with $f\left(y_{n}\right)=y_{n-1}$. Thus $f\left(y_{1}\right)=$ $f^{2}\left(y_{2}\right)=\cdots=f^{n}\left(y_{n}\right)=x$. Since $x \in \operatorname{Ker} f, f(x)=f\left(f^{n}\left(y_{n}\right)\right)=0$, that is, $f^{n+1}\left(y_{n}\right)=0$. So $y_{n} \in \operatorname{Ker} f^{n+1}=\operatorname{Ker} f^{n}$. Consequently, $f^{n}\left(y_{n}\right)=0$ and hence $x=0$, as required.

Lemma 3.6. A quasidiscrete module $M$ is discrete if and only if $M$ is a WH module.

Proof. The necessity of the condition is obvious. Conversely, let $f: M \rightarrow N$ be an epimorphism, with kernel $K$, onto the direct summand $N$ of $M$. As $M$ is quasidiscrete, there is 
a decomposition $M=A \oplus B$ with $A \leq K$ and $B \cap K \ll B$. Now, $N \simeq M / K=K+B / K \simeq$ $B /(B \cap K)$. Theorem 4.24 in [4] yields $N \simeq B$, let $g: N \rightarrow B$ be an isomorphism. Then,

$$
M=A \oplus B \longrightarrow A \oplus N \longrightarrow A \oplus B=M
$$

is an epimorphism with the small kernel $B \cap K$, where $\left.1 \oplus f\right|_{B}: M \rightarrow A \oplus N$ and $1 \oplus g$ : $A \oplus N \rightarrow A \oplus B=M$. By assumption, it is an isomorphism, that is, $B \cap K=0$. Then $K=A$ is a direct summand of $M$ and $f$ splits. This completes the proof.

A discrete module is a quasidiscrete module, but a quasidiscrete module need not be a discrete module. Thus the following result gives a sufficient condition such that a quasidiscrete module is a discrete module.

Corollary 3.7. Let $M$ be a module with ACC on small submodules. Then $M$ is quasidiscrete if and only if $M$ is discrete.

Proof. It follows from Theorem 3.5 and Lemma 3.6.

Proposition 3.8. Let $P$ be a property of modules preserved under isomorphism. If a module $M$ has the property $P$ and satisfies ACC on nonzero small submodules $N$ such that $M / N$ has the property $P$, then $M$ is $W H$.

Proof. Suppose that $M$ is not WH. Then there exists a submodule $N_{1}$ with $N_{1} \neq 0$ and $M / N_{1} \simeq M$. Thus $M / N_{1}$ is not WH but satisfies $P$. Hence, there exists a submodule $N_{2} \leq$ $N_{1}$ with $N_{1} \neq N_{2}$ and $M / N_{2} \simeq M / N_{1}$. So we get $0<N_{1}<N_{2}$ with $M / N_{i} \simeq M$ for $i=1,2$. Repeating the process yields a chain of submodules of the type that contradicts our hypothesis.

Corollary 3.9. If $M$ is $G C H$ and satisfies ACC on small submodules $N$ such that $M / N$ is $G C H$, then $M$ is $W H$.

Proof. It follows by Proposition 3.8.

Remark 3.10. Following Proposition 2.7, if a module $M$ is WH and has DCC on essential WH submodules, then $M$ is GCH.

Corollary 3.11. If $M$ has ACC on its nonzero small submodules $N$ such that $M / N$ is not WH, then $M$ is $W H$.

Proof. Let $P$ be the property of being WH, and suppose that $M$ is not WH. By Proposition 3.8, $M$ must be WH. This contradiction proves that $M$ is $\mathrm{WH}$.

Example 3.12. Let $M$ be a torsion-free Abelian group of finite rank such that $\operatorname{End}(M)$ is a principal ideal domain and let $B=\oplus_{n} M$, where $n$ is an integer. Then the kernel of endomorphism of $B$ is a direct summand which, again, is a direct sum of copies of $M$. It is clear to see that $M$ is $\mathrm{WH}$, but it need not be Hopfian.

Example 3.13. If $R$ is a semisimple Artinian ring, then a module $M$ is Hopfian if and only if $M$ has finite length (see [3]). Note that a vector space $V$ over a field $F$ is Hopfian if and only if it is finite dimensional. Thus an infinite-dimensional vector space is $\mathrm{WH}$, but it is not Hopfian. 


\section{Generalizations of Hopfian and co-Hopfian modules}

Let $R$ be a ring and $\mathscr{C}$ a class of $R$-modules, we will say that $\mathscr{C}$ is socle fine whenever for any $M, N \in \mathscr{C}$, we have $\operatorname{Soc}(M) \simeq \operatorname{Soc}(N)$ if and only if $M \simeq N$.

THeOREM 3.14. For any ring $R$, the following statements are equivalent.

(1) $R$ is semisimple.

(2) The class of all GCH modules is socle fine.

(3) The class of all WH modules is socle fine.

Proof. It is easy to prove that quasiprojective (quasiinjective) and semisimple modules are $\mathrm{GCH}(\mathrm{WH})$ modules. If $R$ is semisimple, then the class of all $R$-modules is socle fine and hence (1) implies (2) and (3).

$(2) \Rightarrow(1)$ Since $R$ and $\operatorname{Soc}(R)$ are quasiprojective and $\operatorname{Soc}(R)=\operatorname{Soc}(\operatorname{Soc}(R))$, we have $R \simeq \operatorname{Soc}(R)$ by (2). Thus $R$ is semisimple.

(3) $\Rightarrow$ (1) Since $\operatorname{Soc}(E(R))=\operatorname{Soc}(R)=\operatorname{Soc}(\operatorname{Soc}(R))$ and both of $E(R)$ and $\operatorname{Soc}(R)$ are quasiinjective, we have $E(R) \simeq \operatorname{Soc}(R)$ is semisimple, and so is $R$.

\section{References}

[1] A. Ghorbani and A. Haghany, Generalized Hopfian modules, J. Algebra 255 (2002), no. 2, 324341.

[2] A. Haghany and M. R. Vedadi, Modules whose injective endomorphisms are essential, J. Algebra 243 (2001), no. 2, 765-779.

[3] V. A. Hiremath, Hopfian rings and Hopfian modules, Indian J. Pure Appl. Math. 17 (1986), no. 7 , 895-900.

[4] S. H. Mohamed and B. J. Müller, Continuous and Discrete Modules, London Mathematical Society Lecture Note Series, vol. 147, Cambridge University Press, Cambridge, 1990.

[5] K. Varadarajan, On a theorem of Shock, Comm. Algebra 10 (1982), no. 20, 2205-2222.

[6] R. Wisbauer, Foundations of Module and Ring Theory, Algebra, Logic and Applications, vol. 3, Gordon and Breach Science Publishers, Pennsylvania, 1991.

Yongduo Wang: Department of Applied Mathematics, Lanzhou University of Technology, Lanzhou 730050, China

E-mail address: ydwang333@sohu.com 


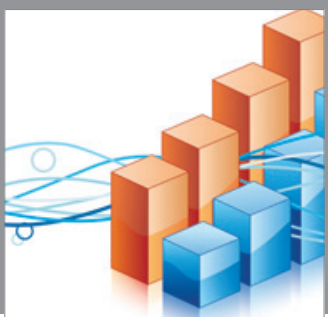

Advances in

Operations Research

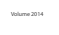

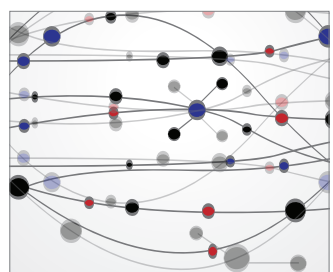

\section{The Scientific} World Journal
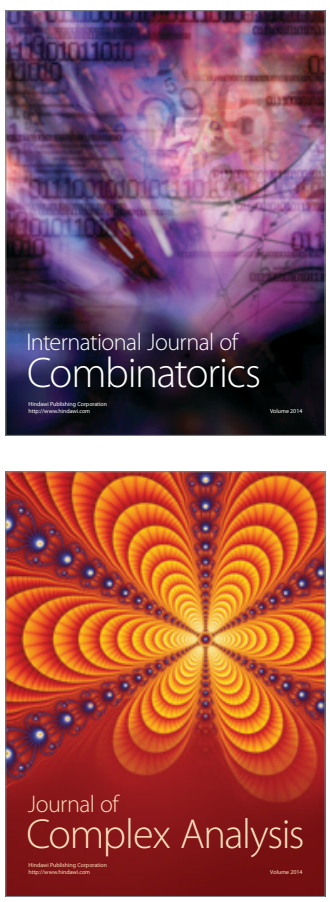

International Journal of

Mathematics and

Mathematical

Sciences
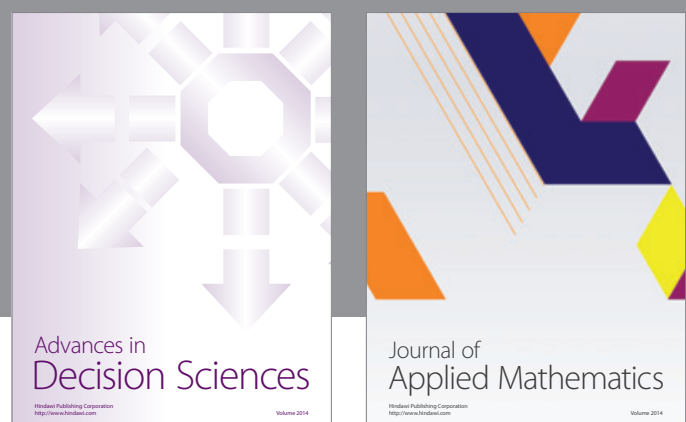

Journal of

Applied Mathematics
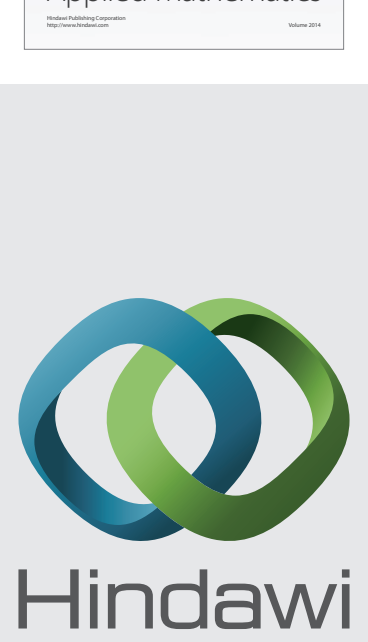

Submit your manuscripts at http://www.hindawi.com
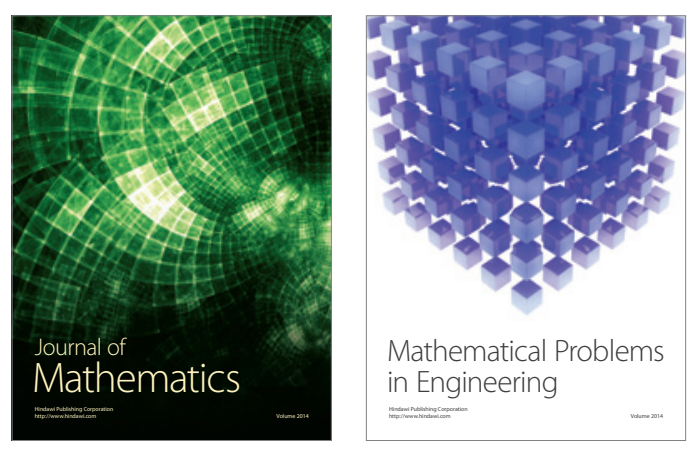

Mathematical Problems in Engineering
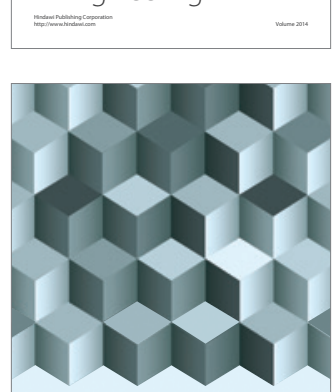

Journal of

Function Spaces
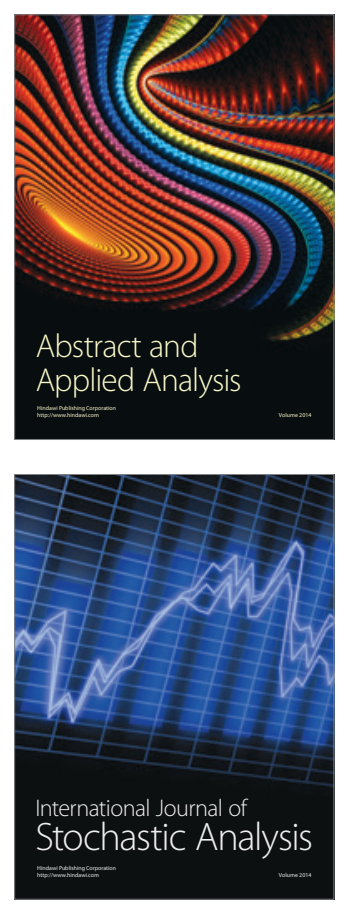

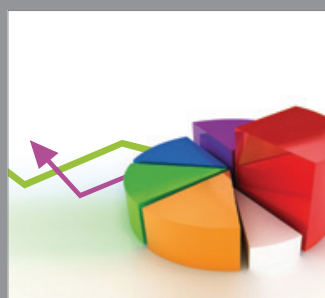

ournal of

Probability and Statistics

Promensencen
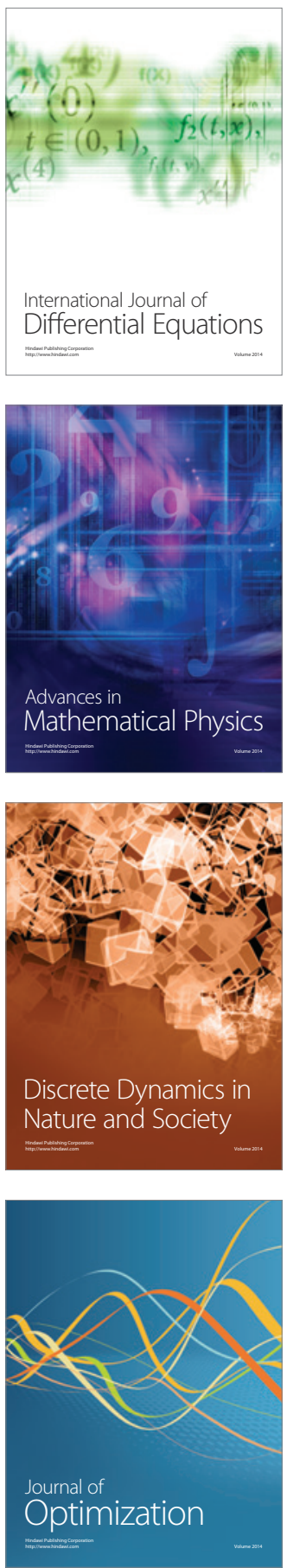